\title{
Mrk 1087: a puzzling suspected Wolf-Rayet galaxy
}

\author{
César Esteban, Ángel R. López-Sánchez \\ Instituto de Astrofísica de Canarias, \\ C/ Vía Láctea s/n, E-38200 La Laguna, Tenerife, España \\ Mónica Rodríguez \\ Instituto Nacional de Astronomía, Óptica y Electrónica, \\ Apdo. Postal 51 y 216, 72000 Puebla, Pue., México
}

\begin{abstract}
We present new observations of Mrk 1087 that indicate this galaxy is experiencing a strong star formation event due to the interaction with -at least- two nearby galaxies, one of them is a star-forming dwarf. We also discover that some of the surrounding non-stellar objects are tidal dwarfs hosting weak starbursts.
\end{abstract}

\section{Observations}

Mrk $1087(d=111 \mathrm{Mpc})$ is a suspected Wolf-Rayet galaxy (Schaerer et al. 1999). It has a companion galaxy (K72 103a) at $81 \mathrm{kpc}$ to the southwest and at the same distance. Keel (1988) proposes that the object is a galaxy in formation surrounded by filaments of infalling ionized gas. We have obtained long-slit intermediate-resolution spectroscopy covering most of the objects surrounding the galaxy, as reported by Méndez \& Esteban (2000), and a deep $V$-band CCD image of the whole group (Figure 1). Our new image shows a new faint companion galaxy $\left(\mathrm{M}_{V} \simeq-18.2\right)$ just to the north of Mrk 1087 (labeled as companion in Figure 1). It has an elliptical shape and shows nebular emission. It shows a radial velocity of $+117 \mathrm{~km} \mathrm{~s}^{-1}$ with respect to the main body of Mrk 1087 . Therefore, both galaxies may be physically associated and at a relative distance of about $60 \mathrm{kpc}$.

\section{Results}

We have detected faint nebular emission in objects \#1, \#3, and \#7, but not in \#12. Objects \#2 and \#11 were not observed. Objects \#1 and \#3 show radial velocities close to the systemic velocity of Mrk 1087. Keel (1988) interpreted \#7 as a companion external object, interacting with the main galaxy. However, we do not confirm his result; in fact \#7 seems to follow closely the general rotation pattern of the ionized gas of the main galaxy. We do not detect the [O III] $4363 \AA$ line in any of the spectra and, therefore, we have estimated the abundances from the so-called empirical calibrations, making use of the $R_{23}$ parameter and/or the $[\mathrm{N} \mathrm{II}] / \mathrm{H} \alpha$ ratio. The absolute magnitudes indicate, that all the objects except the main body of Mrk 1087 can be considered as dwarf galaxies. The $\mathrm{O} / \mathrm{H}$ ratios estimated for objects \#1 and \#3 are similar to that of 


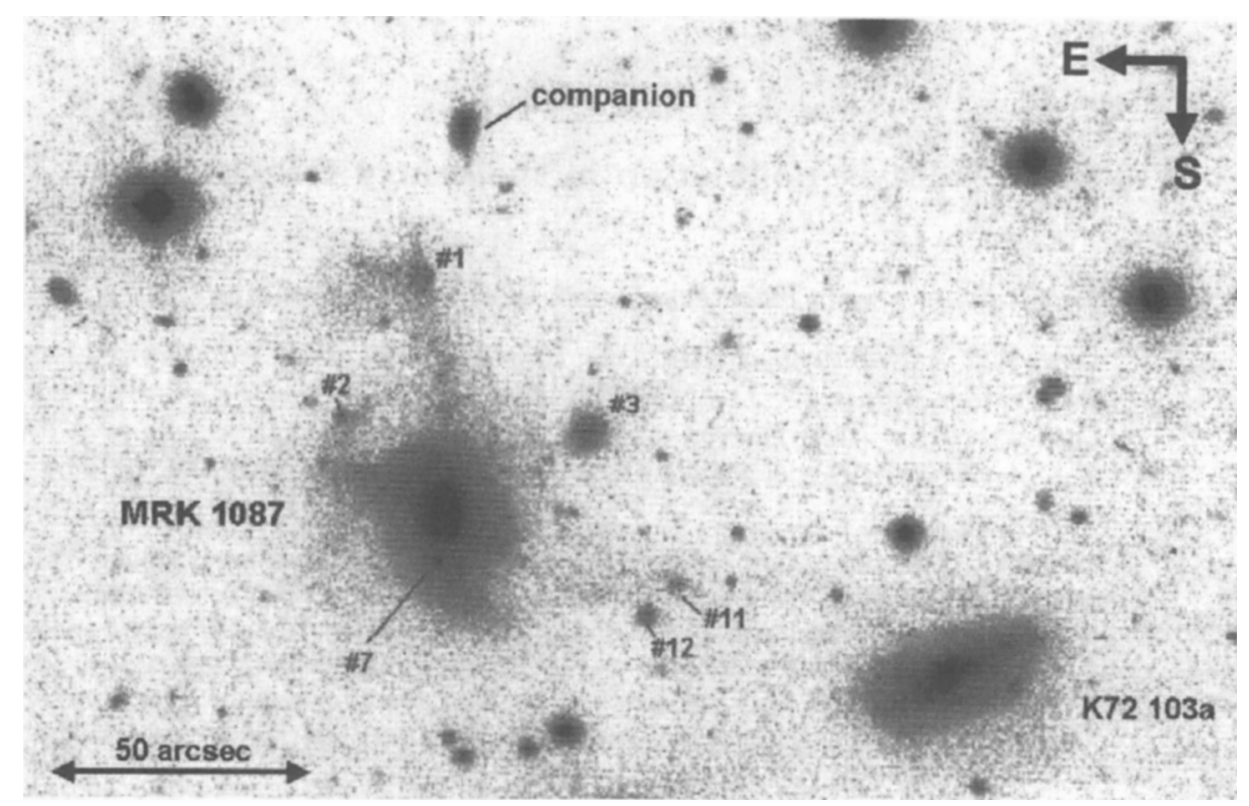

Figure 1. Deep $V$-band CCD image of Mrk 1087 and its surroundings.

the main galaxy $(12+\log (\mathrm{O} / \mathrm{H})=8.7)$ and larger than the abundance expected for the metallicity-luminosity relation for classical dwarfs. This result indicates, that objects \#1 and \#3 can be considered as tidal dwarfs originating from material stripped from Mrk 1087. Object \#7 shows $\mathrm{O} / \mathrm{H}$ and $\mathrm{N}^{+} / \mathrm{O}^{+}$ratios lower but not so different to that of the main galaxy. The $\mathrm{O} / \mathrm{H}$ abundance is larger than that expected for a dwarf galaxy and therefore we interpret \#7 as an intense star-forming zone off-center of $\mathrm{Mrk} 1087$. The $\mathrm{O} / \mathrm{H}$ ratio obtained for the companion object $(12+\log (\mathrm{O} / \mathrm{H})=8.3)$ is lower than that of the main galaxy and consistent with the metallicity-luminosity relation for dwarfs. Moreover, the $\mathrm{N}^{+} / \mathrm{O}^{+}$is also lower than that of Mrk 1087 and of the order of the typical value for dwarf galaxies $(-1.55$, for the main galaxy is -0.99$)$. Therefore, the north companion should be interpreted as an independent nearby dwarf galaxy, that is probably interacting with Mrk 1087. This could explain the presence - at least - of the tidal dwarf \#1 and its associated bridge, which is almost aligned with the companion galaxy (see Figure 1).

In conclusion, we consider that Mrk 1087 and its surroundings can be considered as a compact group of galaxies. This result is a further indication of the importance of the interactions with difficult-to-detect dwarf galaxies in the triggering of strong starbursts, and specially in Wolf-Rayet galaxies.

\section{References}

Keel, W.C. 1988, A\&A 202, 41

Méndez, D.I., Esteban, C. 2000, A\&A 359, 493

Schaerer, D., Contini, T., Pindao, M. 1999, A\&AS 335, 85 